\title{
Extract of Rhizoma Polygonum cuspidatum reduces early renal podocyte injury in streptozotocin-induced diabetic rats and its active compound emodin inhibits methylglyoxal-mediated glycation of proteins
}

\author{
EUNJIN SOHN, JUNGHYUN KIM, CHAN SIK KIM, KYUHYUNG JO and JIN SOOK KIM \\ Korean Medicine Based Herbal Drug Development Group, Herbal Medicine Research Division, \\ Korea Institute of Oriental Medicine, Daejeon 305-811, Republic of Korea
}

Received October 31, 2014; Accepted July 23, 2015

DOI: $10.3892 / \mathrm{mmr} .2015 .4214$

\begin{abstract}
Podocyte injury contributes to renal damage and, eventually, to the occurrence of proteinuria in diabetic nephropathy. The aim of the present study was to investigate the effect of an ethanol extract from Rhizoma Polygonum cuspidatum (P. cuspidatum) on proteinuria and podocyte injury, and elucidate the underlying mechanism for streptozotocin (STZ)-induced diabetic nephropathy. The protective effects of $P$. cuspidatum extract (PCE) on renal podocytes in STZ-induced diabetic rats were also investigated. PCE (100 or $350 \mathrm{mg} / \mathrm{kg} /$ day) was administered to STZ-induced diabetic rats for 16 weeks, and blood glucose levels, body weight and proteinuria were measured. A double labeling technique with the terminal deoxynucleotidyl transferase dUTP nick end labeling assay was performed and synaptopodin expression was observed. In addition, cleaved caspase-3, methylglyoxal (MGO) and 8-hydroxydeoxyguanosine (8-OHdG) expression levels were measured. STZ-induced diabetic rats developed hyperglycemia and proteinuria. Increased apoptosis of the podocytes and increased cleaved caspase-3, MGO and 8-OHdG expression levels, as well as decreased synaptopodin expression were detected in the glomeruli of STZ-induced diabetic rats. However, treatment with PCE for 16 weeks restored protein levels to normal, and reduced podocyte loss and apoptosis. Levels of caspase-3 and MGO expression, as well as oxidative stress were ameliorated by PCE treatment. In addition, emodin, a biologically active ingredient of PCE, exerted an MGO scavenging effect and inhibited MGO-derived advanced glycation end-product formation. These findings indicate that
\end{abstract}

Correspondence to: Dr Jin Sook Kim, Korean Medicine Based Herbal Drug Development Group, Herbal Medicine Research Division, Korea Institute of Oriental Medicine, 1672 Yuseongdae-ro, Daejeon 305-811, Republic of Korea

E-mail: jskim@kiom.re.kr

Key words: diabetic nephropathy, podocyte loss, oxidative stress, methylglyoxal, podocyte injury
PCE may be administered to prevent proteinuria and podocyte loss in STZ-induced diabetic rats partly by inhibiting podocyte apoptosis and cleaved caspase-3 expression, and by restoring the balance of oxidative stress and MGO expression.

\section{Introduction}

It is well known that diabetes-induced renal disease is a common complication for diabetes patients, leading to end-stage renal failure (1). Studies in patients with diabetes have demonstrated that podocyte loss and depletion represent a few of the early pathogenic mechanisms of diabetic nephropathy $(2,3)$. Podocytes are highly specialized cells that localize on the outer surface of the glomerular basement membrane, and are important in maintaining the function and structure of the glomerular filtration barrier (4). Thus, podocyte injury inevitably results in proteinuria (5), as clinically, glomerular proteinuria is caused by structural and functional abnormalities in the glomerular filtration barrier (6). Furthermore, increased levels of oxidative stress are observed in diabetes in vivo and in vitro $(7,8)$. Podocyte injury is a major contributor to the pathogenesis of diabetic nephropathy via the excessive generation of oxidative stress (9).

Methylglyoxal (MGO), a reactive dicarbonyl intermediate, is a potent precursor of advanced glycation end-products (AGEs) and there is increasing evidence demonstrating that MGO levels in diabetic patients are elevated $(10,11)$. MGO is highly cytotoxic and forms stable adducts, and in the plasma of diabetic patients and diabetic animal tissues its concentration levels have been observed to be markedly higher $(12,13)$. MGO is hypothesized to cause podocyte damage, and the MGO-induced generation of reactive oxygen species may elicit apoptosis $(14,15)$. Our previous study demonstrated that MGO-induced oxidative stress may be involved in podocyte apoptosis in diabetic nephropathy (16).

Medicinal herbs have been administered for hundreds of years as a folk medicine to treat diabetic complications and diabetes (17,18). Rhizoma Polygonum cuspidatum (P. cuspidatum) is the dried rhizome of P. cuspidatum Sieb. et Zucc. (Polygonaceae). This herb has been administered to control oral diseases in patients in Korea (19), and has been used as 
a medicinal herb to treat numerous diseases and disorders in Asian countries, including inflammatory diseases and Korea, China and Japan (20). Additionally, P. cuspidatum has been widely administered as a medicinal herb for anti-diabetic effects in traditional Chinese medicine (21).

However, $P$. cuspidatum exerts unknown effects on diabetic complications. Therefore, the preventative effect of the ethanol extract from $P$. cuspidatum on early renal injury in streptozotocin (STZ)-induced diabetic rats was investigated

\section{Materials and methods}

Preparation of P. cuspidatum extract (PCE). Rhizoma P. cuspidatum was commercially obtained from Jung-dong, Daejeon, Korea in November 2008. The dried and ground plant material $(6.8 \mathrm{~kg})$ was extracted with ethanol (108 liters) by maceration at room temperature for three days, and the extracts were combined and concentrated in vacuo at $40^{\circ} \mathrm{C}$ to provide $580 \mathrm{~g}$ lyophilized PCE. A voucher specimen was deposited in a herbarium at the Korea Institute of Oriental Medicine (KIOM; Daejeon, Korea).

Induction of diabetic rats and experimental design. All experiments were conducted according to the National Institutes of Health (NIH) Guide for the Care and Use of Laboratory Animals and were approved by the KIOM Institutional Animal Care and Use Committee (22). Seven-week-old male Sprague-Dawley rats were maintained in an automatically controlled room (temperature, $\sim 24^{\circ} \mathrm{C}$; humidity, $60 \%$ ) under conventional lighting. The rats were injected with STZ $(60 \mathrm{mg} / \mathrm{kg}$ i.p.; Sigma-Aldrich, St. Louis, MO, USA). Control rats received a vehicle $(0.01 \mathrm{M}$ citrate buffer, $\mathrm{pH} 4.5$ ). One week after the STZ injection, a blood sample (100-200 $\mu \mathrm{l})$ was obtained from the tail vein. The rats (blood glucose level $>300 \mathrm{mg} / \mathrm{dl}$ ) were randomly assigned to four groups $(n=8)$. PCE was dissolved in a vehicle $(0.5 \% \mathrm{w} / \mathrm{v}$ carboxymethyl cellulose solution; Sigma-Aldrich) at a concentration of $50 \mathrm{mg} / \mathrm{ml}$. Two groups of STZ-induced diabetic rats received a daily gastric gavage of PCE (100 or $350 \mathrm{mg} / \mathrm{kg}$ ) and the other groups were administered the same quantity of vehicle gavage for 16 weeks. Rat body weights and blood glucose levels were monitored one a week.

Metabolic and physical analysis. When the rats reached 25 weeks of age, fasting blood glucose was measured using an automated Hitachi912 chemistry analyzer (Hitachi, Ltd., Tokyo, Japan). A 24-h urine sample (1 ml) was obtained from the rats using a metabolic cage and the proteinuria levels were measured using a competitive enzyme-linked immunosorbent assay (ELISA) kit (UP ELISA kits; NeoScientific, Ltd., Cambridge, MA, USA).

Quantification of 8-hydroxydeoxyguanosine (8-OHdG) in the urine and $M G O$ in the renal cortex. To investigate induced oxidative stress by $\mathrm{MGO}$ in the diabetic rats, urine levels of 8-OHdG were measured using a competitive ELISA kit (JaICA, Shizuoka, Japan). MGO levels in the renal cortex were analyzed using an MGO adduct (Cosmo Bio Co., Tokyo, Japan), as previously described (16).

Immunofluorescent staining and immunohistochemistry. Immunohistochemistry was performed as previously described (15). Briefly, at the end of the experimental period, the rats were anesthetized with pentobarbital sodium $(60 \mathrm{mg} / \mathrm{kg}$, i.p; Hanlim Pharmaceutical Company, Seoul, Korea) and sacrificed. Following removal of the kidneys, part was flash frozen in liquid nitrogen and stored at $-80^{\circ} \mathrm{C}$ until analysis and another part was fixed (4\% paraformaldehyde) for histological analysis. The renal sections ( $4 \mu \mathrm{m}$ thick) were deparaffinized and hydrated by sequential immersion in xylene and graded alcohol solutions (Sigma-Aldrich) and then treated with normal serum from the same species that contained the secondary antibody to block non-specific staining (Abcam, Cambridge, MA, USA). The slides were incubated overnight at $4^{\circ} \mathrm{C}$ with the primary antibody. The primary antibodies were anti-mouse monoclononal MGO (1:500; cat. no. NOF-N213430-EX; CosmoBio Co, Ltd., Toyko, Japan), anti-rabbit polyclonal synaptopodin and anti-mouse monoclonal 8-OHdG, (1:500; cat. nos. sc-50459 and sc-393870, Santa Cruz Biotechnology, Inc., Dallas, TX, USA), anti-rabbit polyclonal WT-1 (1:500; cat. no. AF-5729; R\&D Systems, Inc., Minneapolis, MN, USA) and anti-rabbit polyclonal cleaved caspase-3 (1:500; cat. no. 9661; Cell Signaling Technology, Inc., Danvers, MA, USA). To detect 8-OHdG and cleaved caspase-3, sections were incubated with an Envision ${ }^{\mathrm{TM}}$ kit (Dako North America, Inc., Carpinteria, CA, USA) and visualized using 3,3'-diaminobenzidine tetrahydrochloride (DAB; Vector Laboratories, Inc., Burlingame, CA, USA). The MGO specimens were visualized using a red alkaline phosphatase substrate kit (Vector Laboratories, Inc.). To detect Wilms Tumor 1 (WT-1; a known podocyte marker) $(23,24)$ and synaptopodin, the sections were incubated with rhodamine-labeled anti-rabbit immunoglobulin (Ig)G (Santa Cruz Biotechnology, Inc.) and fluorescein isothiocyanate-labeled anti-mouse IgG (Santa Cruz Biotechnology, Inc.), respectively, and visualized by fluorescence microscopy (BX51; Olympus Corporation, Tokyo, Japan). Immunohistochemical negative controls were performed by incubating the section with rat normal serum. For morphometric analysis, the positive cell number and positive staining per individual glomerulus in a total of 50 glomeruli were determined using ImageJ software (v1.46r; NIH, Bethesda, MD, USA).

Double labeling technique for terminal deoxynucleotidyl transferase dUTP nick end labeling (TUNEL) and synaptopodin expression levels. TUNEL staining was performed using a DeadEnd ${ }^{\mathrm{TM}}$ Colorimetric TUNEL system kit (Promega Corporation, Madison, WI, USA) according to the manufacturer's instructions. Apoptotic cells were detected with a brown colored solution containing peroxidase substrate and the stable chromogen, DAB. Labeling with anti-synaptopodin (Santa Cruz Biotechnnology, Inc.) was performed on the same section with secondary detection using an alkaline phosphatase-conjugated anti-mouse IgG and a red alkaline phosphatase substrate kit (Vector Laboratories, Inc.). To prevent a cross-reaction with the dual labeling procedure, the slide was incubated with normal rat serum for $1 \mathrm{~h}$ at $37^{\circ} \mathrm{C}$ following TUNEL staining. Counterstaining was performed with hematoxylin (Vector Laboratories, Inc.). For morphometric analysis, the positive signal for synaptopodin was measured using ImageJ software and the cells that were dually labeled for TUNEL and synaptopodin were counted under a microscope (BX51; Olympus Corporation). 
Table I. Metabolic and physical parameters of the rats.

\begin{tabular}{lcccc}
\hline Parameter & NOR & DM & PCE-100 & PCE-350 \\
\hline Blood glucose, mmol/1 & $9.00 \pm 0.60$ & $32.62 \pm 2.41^{\mathrm{a}}$ & $29.46 \pm 3.22$ & $26.34 \pm 4.91$ \\
Body weight, g & $475.9 \pm 10.70$ & $208.5 \pm 12.10^{\mathrm{a}}$ & $205.5 \pm 23.10$ & $206.0 \pm 16.40$ \\
Proteinuria, mg/day & $7.42 \pm 2.14$ & $16.37 \pm 3.89^{\mathrm{a}}$ & $13.67 \pm 2.56$ & $11.54 \pm 3.16^{\mathrm{b}}$ \\
\hline
\end{tabular}

NOR, normal rat; DM, streptozotocin-induced diabetic rat; PCE-100, DM treated with $100 \mathrm{mg} / \mathrm{kg}$ PCE; PCE-350, DM treated with $350 \mathrm{mg} / \mathrm{kg}$ PCE. All data are expressed as means \pm standard error of the mean $(\mathrm{n}=8) .{ }^{\mathrm{a}} \mathrm{P}<0.01 \mathrm{vs}$. NOR group; ${ }^{\mathrm{b}} \mathrm{P}<0.01$ vs. DM group. PCE, Polygonum cuspidatum extract.

Western blot analysis. Renal cortex tissues were homogenized in solutions containing $250 \mathrm{mM}$ sucrose, $1 \mathrm{mM}$ ethylenediaminetetraacetic acid (Sigma-Aldrich), $0.1 \mathrm{mM}$ phenylmethylsulfonyl fluoride (Sigma-Aldrich) and $20 \mathrm{mM}$ potassium phosphate buffer ( $\mathrm{pH}$ 7.6; Sigma-Aldrich). Proteins $(20 \mu \mathrm{g})$ were subjected to immunoblotting onto polyvinylidene fluoride (PVDF) membranes (Bio-Rad Laboratories, Inc.) and the PVDF membranes were incubated with the following primary antibodies: Cleaved caspase-3 $(1: 1,500)$ and $\beta$-actin (1:3,000; Sigma-Aldrich) at $4^{\circ} \mathrm{C}$ overnight. The bound horseradish peroxidase-conjugated secondary antibody was detected using an enhanced chemiluminescence detection system (Intron Biotechnology, Inc., Seoul, Korea). The protein expression levels were determined by analyzing the signals captured on the PVDF membranes with an image analyzer (Las-3000; Fuji Photo Film Co., Ltd., Tokyo, Japan).

MGO-modified bovine serum albumin (BSA) formation assay. An MGO-BSA assay was performed with certain modifications, as previously described (25). BSA (10 mg/ml) was incubated with MGO $(5 \mathrm{mM})$ in the presence or absence of emodin (Sigma-Aldrich) in phosphate-buffered saline (100 mM; pH 7.4; Biosesang, Inc., Sungnam, Korea) at $37^{\circ} \mathrm{C}$ for seven days. Emodin was diluted with $1 \%$ dimethyl sulfoxide (Sigma-Aldrich) and aminoguanidine (AG; Sigma-Aldrich) served as a positive control. After seven days, the fluorescence of the sample was measured using a Synergy ${ }^{\mathrm{TM}}$ HT, Gen5 ${ }^{\mathrm{TM}}$ fluorescence microplate reader (Bio-Tek Instruments, Inc., Winooski, VT, USA) at emission and excitation wavelengths of 450 and $360 \mathrm{~nm}$, respectively. All measurements were performed in triplicate.

MGO scavenging assay. A fluorogenic assay for MGO was performed as previously described (26). A potential fluorogenic probe, 4,5-diaminofluorescein (DAF-2), which reacts with MGO, exhibits a small shift in the fluorescence wavelength and a marked increase in fluorescence intensity for the probe-MGO adduct. DAF-2 [100 $\mu \mathrm{l}(100 \mu \mathrm{M})$; Sigma-Aldrich) was incubated with $50 \mu \mathrm{l} \mathrm{MGO}(2 \mathrm{mM})$ in $10 \mathrm{mM}$ phosphate-buffered saline ( $\mathrm{pH} 7.4)$ at $37^{\circ} \mathrm{C}$ and the fluorescence spectra values were obtained at 0,1 and $2 \mathrm{~h}$. The different florescence characteristic of the isomeric mixture of the MGO-DAF-2 adduct developed and maximized within $1 \mathrm{~h}$. The excitation and emission wavelengths were 435 and $509 \mathrm{~nm}$, respectively. Emodin and AG were serially diluted with the MGO-DAF-2 mixture and each final concentration was $5 \mathrm{mg} / \mathrm{ml}$ in phosphate-buffered saline
( $\mathrm{pH}$ 7.4). To accommodate the minimum volume requirement for the microplate used in the current study, $100 \mu \mathrm{l} \mathrm{DAF-2}$ solution, $50 \mu \mathrm{l}$ MGO solution, and $50 \mu \mathrm{l}$ emodin and AG solution were used, respectively. AG served as a positive control. The fluorescence of the sample was measured using a Gemini XPS Microplate Reader (Molecular Devices, LLC, Sunnyvale, CA, USA).

Statistical analysis. Data are expressed as the mean \pm standard error of the mean and analyzed using one-way analysis of variance followed by Tukey's multiple comparison test or an unpaired Student's t-test. Prism 6.0 software (Graph pad, San Diego, CA, USA) was used to perform statistical analyses.

\section{Results}

Treatment with PCE reduces proteinuria. At 25 weeks of age, the body weights were significantly decreased and the blood glucose levels were significantly increased in the STZ-induced diabetic rats when compared with the normal control rats $(\mathrm{P}<0.01)$. However, no statistically significant differences in the body weights and blood glucose levels between the STZ-induced diabetic rats and the PCE-treated diabetic rats were observed. The level of proteinuria was significantly increased in the STZ-induced diabetic rats compared with the normal control rats $(\mathrm{P}<0.01)$. However, PCE treatment significantly decreased proteinuria compared with the vehicle-treated diabetic rats in a dose-dependent manner $(\mathrm{P}<0.01$; Table I).

Treatment with PCE ameliorates podocyte loss in the renal glomerulus. The podocyte content was determined by counting the cells that were stained with synaptopodin and WT-1, the podocyte marker (Fig. 1A and B). The number of synaptopodin- and WT-1-positive cells in the STZ-induced diabetic rat group was reduced compared with that of the normal control rats $(\mathrm{P}<0.01)$. Treatment with $\mathrm{PCE}$ markedly increased the podocyte content in the renal glomeruli in a dose-dependent manner $(\mathrm{P}<0.01$; Fig. $1 \mathrm{~B}$ and $\mathrm{C})$.

Treatment with PCE inhibits podocyte apoptosis in the renal glomerulus. To determine the anti-apoptotic effect of PCE, the double labeling technique for TUNEL and synaptopodin was performed. A reduced number of synaptopodin-labeled cells was observed in the glomeruli of STZ-induced diabetic rats compared with the normal control rats $(\mathrm{P}<0.01)$. However, in the PCE-treated diabetic rats, the level of synaptopodin-positive 
A
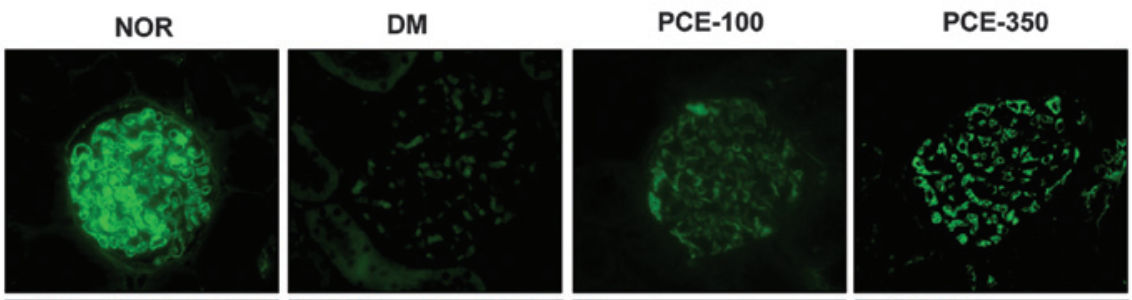

B
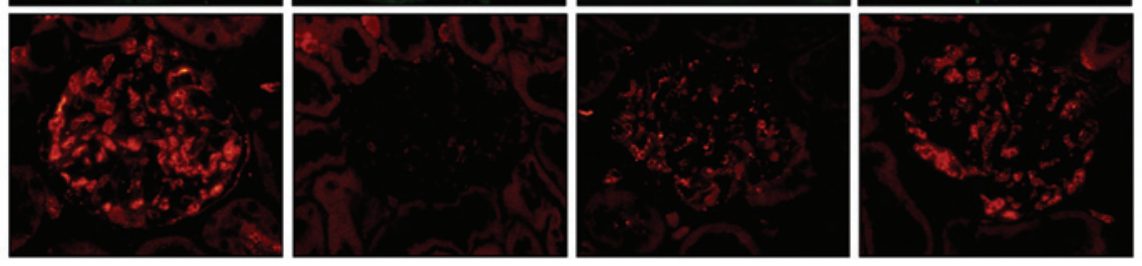

C

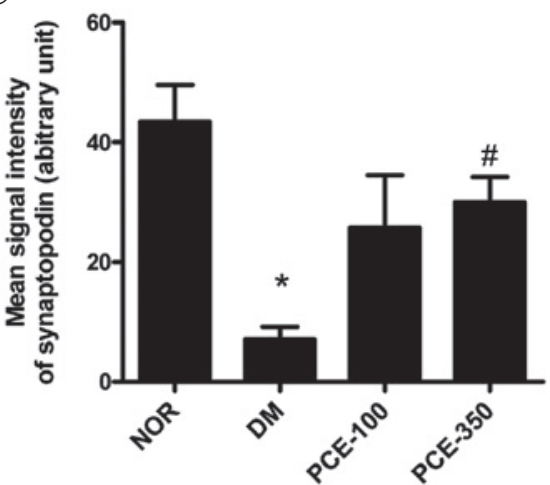

D

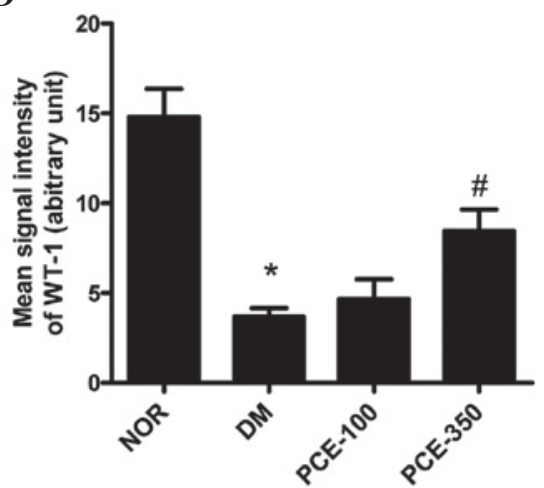

Figure 1. Effect of PCE on podocyte loss. Representative histology sections from the NOR, DM, PCE-100 and PCE-350 groups (magnification, x400): (A) Synaptopodin and (B) WT-1 staining of the glomeruli. Morphometric analysis of (C) synaptopodin and (D) WT-1 in the renal cortex of rats from each group. All data are expressed as the mean \pm standard error of the mean $(\mathrm{n}=8) .{ }^{*} \mathrm{P}<0.01$ vs. NOR group; ${ }^{*} \mathrm{P}<0.01$ vs. DM group. NOR, normal rat; DM, streptozotocin-induced diabetic rat; PCE-100, DM treated with $100 \mathrm{mg} / \mathrm{kg}$ PCE; PCE-350, DM treated with $350 \mathrm{mg} / \mathrm{kg}$ PCE; PCE, Polygonum cuspidatum extract; WT-1, Wilms Tumor 1.

A

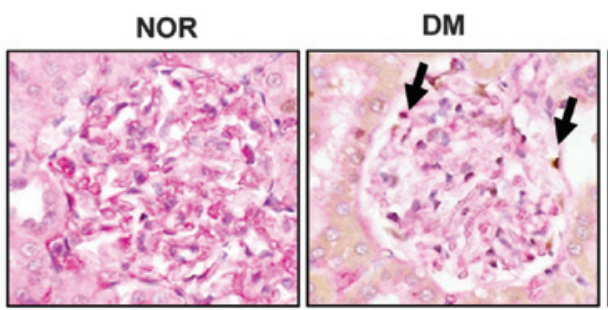

PCE-100

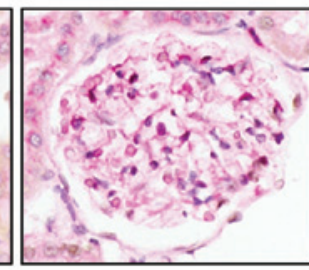

PCE-350

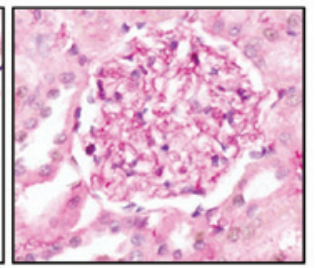

C

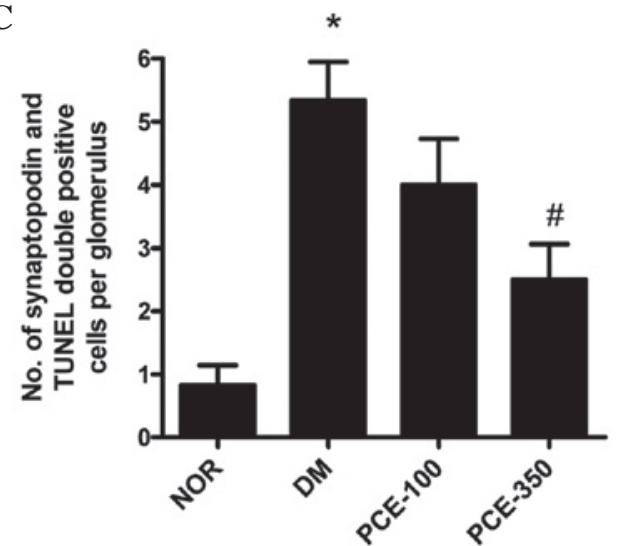

Figure 2. Effect of PCE on the apoptosis of podocytes. (A) Dual-labeling for TUNEL (brown) and synaptopodin (red) in kidney sections from the NOR, DM, PCE-100 and PCE-350 groups (magnification, x400). Morphometric analysis of (B) the positive area of synaptopodin expression and (C) TUNEL and synaptopodin double-positive cells. Hematoxylin (blue) was used to counterstain the nucleus in (A). All data are expressed as the mean \pm standard error of the mean $(\mathrm{n}=8)$. " $\mathrm{P}<0.01$ vs. NOR group; ${ }^{\mathrm{P}} \mathrm{P}<0.01$ vs. DM group. NOR, normal rat; DM, streptozotocin-induced diabetic rat; PCE-100, DM treated with $100 \mathrm{mg} / \mathrm{kg}$ PCE; PCE-350, DM treated with $350 \mathrm{mg} / \mathrm{kg}$; PCE, Polygonum cuspidatum extract; TUNEL, terminal deoxynucleotidyl transferase dUTP nick end labeling. 
A

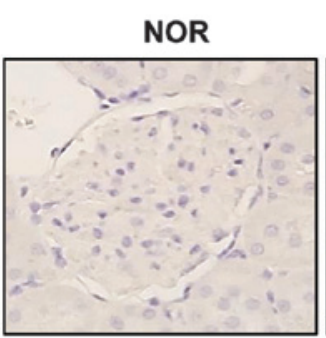

DM

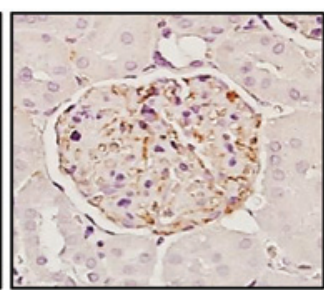

B

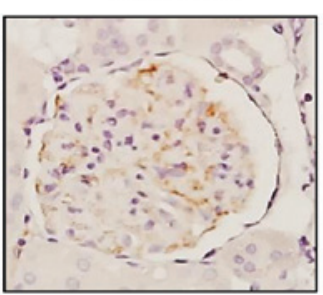

PCE-100

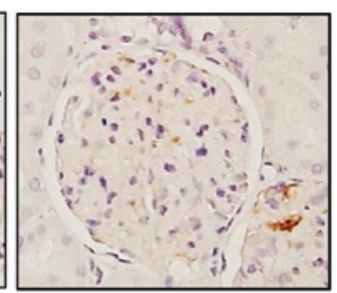

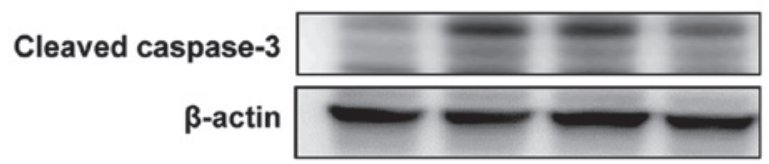

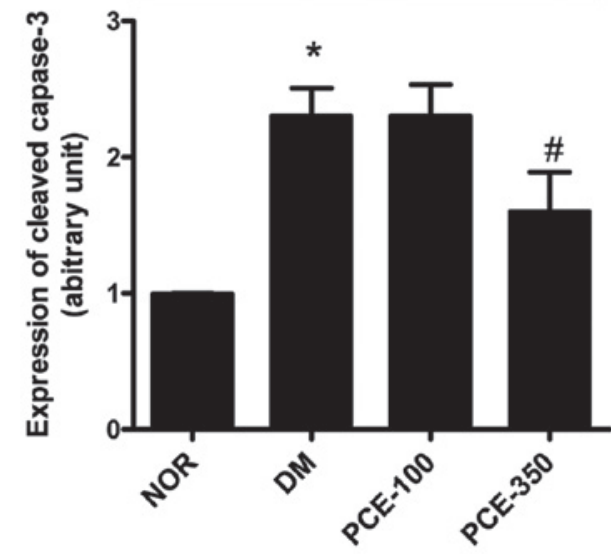

Figure 3. Effect of PCE on caspase-3 expression levels in renal tissue. Representative histology sections from the NOR, DM, PCE-100 and PCE-350 groups (magnification, x400): (A) Immunohistochemical stained cleaved caspase-3 in the glomeruli. (B) Western blot analysis of cleaved caspase-3 in the renal cortex of rats from each group. Data are expressed as the mean \pm standard error of the mean $(\mathrm{n}=8)$. ${ }^{*} \mathrm{P}<0.01$ vs. NOR group; $\mathrm{P}<0.01 \mathrm{vs}$. DM group. NOR, normal rat; DM, streptozotocin-induced diabetic rat; PCE-100, DM treated with $100 \mathrm{mg} / \mathrm{kg}$ PCE; and PCE-350, DM treated with $350 \mathrm{mg} / \mathrm{kg}$ PCE; PCE, Polygonum cuspidatum extract.

A

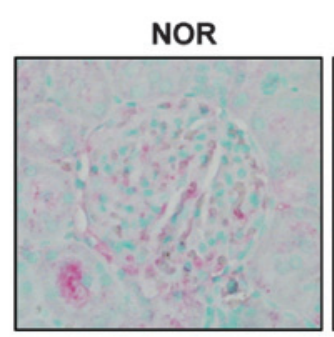

B

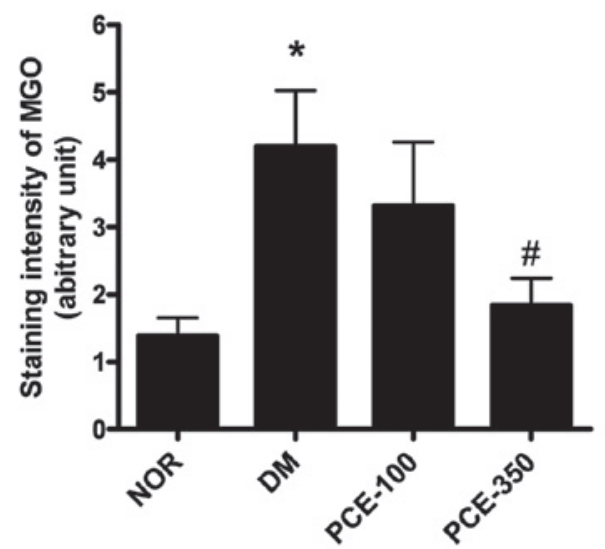

DM

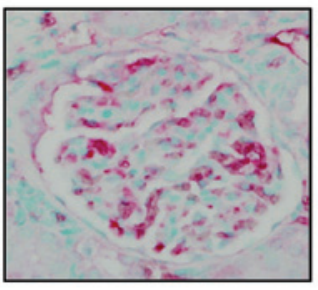

C

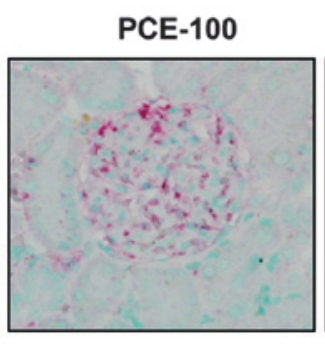

\section{PCE-350}
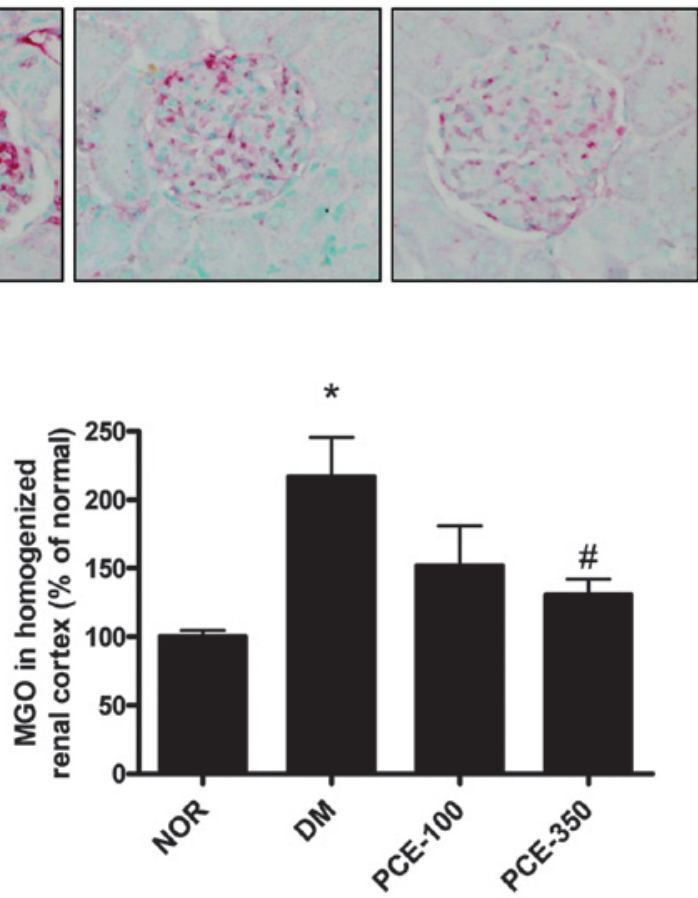

Figure 4. Effect of PCE on the MGO adduct expression levels in renal tissue. (A) MGO staining of the glomeruli in representative histology sections from the NOR, DM, PCE-100 and PCE-350 groups (magnification, x400). Morphometric analysis of MGO levels in the (B) glomerulus and (C) the renal cortex of rats from each group. All data are expressed as the mean \pm standard error of the mean $(\mathrm{n}=8)$. " $\mathrm{P}<0.01$ vs. NOR group; ${ }^{\mathrm{P}}<0.01$ vs. DM group. NOR, normal rat; DM, streptozotocin-induced diabetic rat; PCE-100, DM treated with $100 \mathrm{mg} / \mathrm{kg}$ PCE; PCE-350, DM treated with $350 \mathrm{mg} / \mathrm{kg}$; PCE, Polygonum cuspidatum extract; MGO, methylglyoxal. 
A

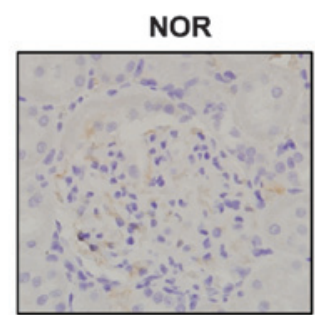

B

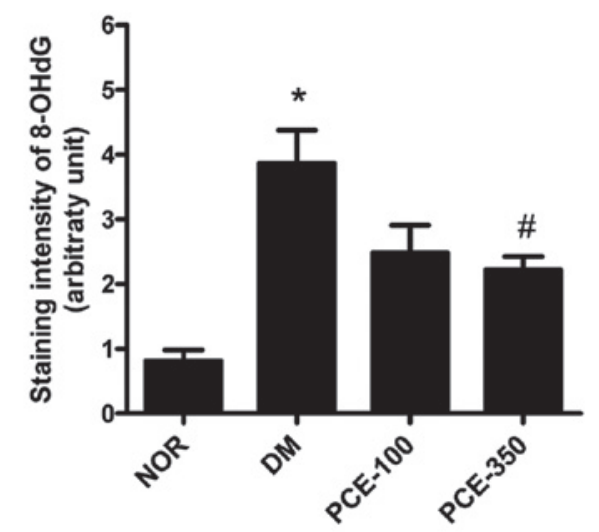

DM

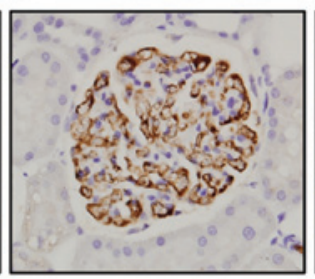

$\mathbf{C}$

C

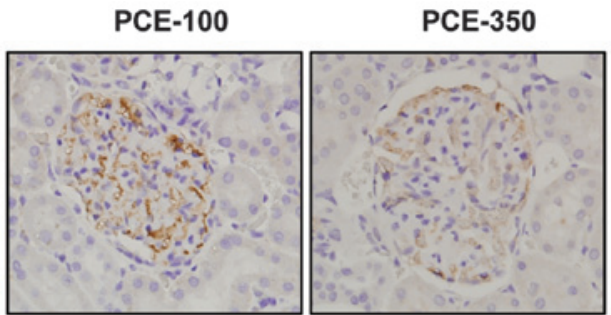

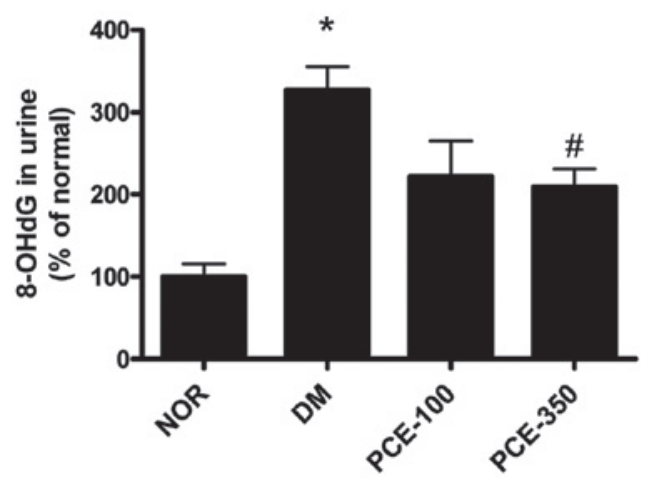

Figure 5. The effect of PCE on 8-OHdG levels in the renal glomeruli and urine. (A) Immunohistochemical staining for 8-OHdG in representative histology sections from the NOR, DM, PCE-100 and PCE-350 groups (magnification, $\mathrm{x} 400$ ). Morphometric analysis of 8-OHdG levels in the (B) glomerulus and (C) urine of rats from each group. All data are expressed as the mean \pm standard error of the mean $(n=8)$. ${ }^{*} \mathrm{P}<0.01$ vs. NOR group; ${ }^{*} \mathrm{P}<0.01$ vs. DM group. NOR, normal rat; DM, streptozotocin-induced diabetic rat; PCE-100, DM treated with $100 \mathrm{mg} / \mathrm{kg}$ PCE; PCE-350, DM treated with $350 \mathrm{mg} / \mathrm{kg}$; PCE, Polygonum cuspidatum extract; 8-OHdG, 8-hydroxydeoxyguanosine.

cells had increased compared with the STZ-induced diabetic rats $(\mathrm{P}<0.01)$. TUNEL-positive cells co-localized to the region of synaptopodin expression in the double labeling-stained kidney section, which is consistent with the apoptosis of podocytes in the STZ-induced diabetic rat glomeruli (Fig. 2A). In the STZ-induced diabetic rats, the number of TUNEL-positive cells was markedly increased compared with that observed in the normal control rats $(\mathrm{P}<0.01)$. $\mathrm{PCE}$ treatment was effective in reducing the apoptotic signal in STZ-induced diabetic rats (Fig. 2B and C).

Treatment with PCE decreases the expression of caspase-3 in renal tissue. To investigate the effect of PCE on cleaved caspase-3 expression in the renal cortex, an immunoassay was performed. The immunohistochemical staining for cleaved caspase-3 demonstrated marked cleaved caspase-3 expression levels in the renal glomeruli of STZ-induced diabetic rats. The expression levels of cleaved caspase-3 were dose-dependently restored in the PCE-treated diabetic rats $(\mathrm{P}<0.01$; Fig. 3A). To validate the immunohistochemistry results, western blot analysis was performed. STZ-induced diabetic rats demonstrated significantly higher levels of caspase-3 compared with those of the normal control rats; furthermore, the increased caspase-3 expression levels in the renal cortex of STZ-induced diabetic rats were reduced by treatment with PCE (Fig. 3B). These results indicate that PCE decreased the cleaved caspase-3 expression levels in the renal cortex of diabetic nephropathy by exerting an anti-apoptotic effect.

PCE treatment attenuates the expression levels of MGO in the glomeruli and the renal cortex. The renal glomeruli of
Table II. Methylglyoxal scavenging activity of emodin.

\begin{tabular}{lcc}
\hline Compound & \% inhibition & $\mathrm{IC}_{50}$ \\
\hline Emodin, $\mu \mathrm{g} / \mathrm{ml}$ & & \\
50 & $83.29 \pm 12.23$ & $112.54 \pm 6.01 \mu \mathrm{g} / \mathrm{ml}$ \\
100 & $52.08 \pm 3.71$ & $(0.41 \pm 2.07 \mathrm{mM})$ \\
250 & $45.82 \pm 2.09$ & \\
Aminoguanidine, $\mu \mathrm{g} / \mathrm{ml}$ & & \\
100 & $71.52 \pm 9.64$ & $116.52 \pm 8.49 \mu \mathrm{g} / \mathrm{ml}$ \\
250 & $38.23 \pm 9.85$ & $(1.05 \pm 1.25 \mathrm{mM})$ \\
500 & $27.49 \pm 5.99$ & \\
\hline
\end{tabular}

Inhibitory activity was expressed as the mean \pm standard error of the mean of triplicate experiments. The $\mathrm{IC}_{50}$ value was calculated from the dose inhibition curve. $\mathrm{IC}_{50}$, half maximal inhibitory concentration.

STZ-induced diabetic rats exhibited significant staining for MGO when compared with the normal control rats $(\mathrm{P}<0.01)$. This increase in MGO staining intensity was reduced in the PCE-treated diabetic rats (Fig. 4A and B). Additionally, in the ELISA analysis, the MGO levels in the homogenized renal cortex were also markedly increased in the STZ-induced diabetic rats. PCE-350 was found to significantly reduce the diabetes-induced increases in MGO level (Fig. 4C).

PCEtreatmentpreventsoxidative stress.Immunohistochemical staining of 8-OHdG expression in the glomeruli demonstrated a significant increase in the STZ-induced diabetic rats 
Table III. Inhibitory effect of emodin on methylglyoxal-derived advanced glycation end-product formation.

\begin{tabular}{lcc}
\hline Compound & \% inhibition & $\mathrm{IC}_{50}$ \\
\hline Emodin, $\mu \mathrm{g} / \mathrm{ml}$ & & $23.81 \pm 2.28 \mu \mathrm{g} / \mathrm{ml}$ \\
5 & $93.22 \pm 2.01$ & $0.08 \pm 0.01 \mathrm{mM}$ \\
10 & $73.49 \pm 1.90$ & \\
25 & $48.44 \pm 2.84$ & \\
Aminoguanidine, $\mu \mathrm{g} / \mathrm{ml}$ & & $787.65 \pm 81.76 \mu \mathrm{g} / \mathrm{ml}$ \\
250 & $97.6 \pm 9.53$ & $7.12 \pm 0.73 \mathrm{mM}$ \\
500 & $70.6 \pm 5.73$ & \\
1,000 & $35.0 \pm 1.36$ & \\
\hline
\end{tabular}

Inhibitory activity was expressed as the mean \pm standard error of the mean of triplicate experiments. The $\mathrm{IC}_{50}$ value was calculated from the dose inhibition curve. $\mathrm{IC}_{50}$, half maximal inhibitory concentration.

compared with the normal control rats $(\mathrm{P}<0.01)$. The $8-\mathrm{OHdG}$ expression level was inhibited by PCE-350 treatment (Fig. 5A and $\mathrm{B}$ ). In addition, the $8-\mathrm{OHdG}$ levels in the urine were markedly increased in the STZ-induced diabetic rats $(\mathrm{P}<0.01)$. However, PCE attenuated these diabetes-induced increases in urine 8-OHdG levels (Fig. 5C).

PCE has an effect on MGO scavenging and inhibition of $M G O-B S A$ formation. PCE contains various flavones as its major chemical constituents, including resveratrol and emodin $(27,28)$. To identify the biological activity of these compounds in PCE against MGO-mediated glycation, MGO scavenging and MGO-BSA formation assays were performed. As shown in Table II, emodin significantly inhibited the probe-MGO adduct when compared with AG, a known positive control. The half maximal inhibitory concentrations $\left(\mathrm{IC}_{50}\right)$ for emodin and $\mathrm{AG}$ were $0.41 \pm 1.25$ and $1.05 \pm 2.07 \mathrm{mM}$, respectively. In addition, emodin significantly inhibited the formation of MGO-derived AGEs and was demonstrated to be more effective than $\mathrm{AG}$, with $\mathrm{IC}_{50}$ values of $0.08 \pm 0.01$ and $7.12 \pm 0.73 \mathrm{mM}$, for emodin and AG, respectively (Table III).

\section{Discussion}

The current study demonstrates that PCE treatment significantly improved certain biochemical markers of renal function, including proteinuria in STZ-induced diabetic rats. The present study revealed that PCE exerts a preventative effect on podocyte loss and apoptosis in the early stages of injury in STZ-induced diabetic rats. In STZ-induced diabetic rats, podocyte apoptosis was associated with the upregulation of cleaved caspase-3 overexpression, which was partially restored to normal levels by PCE treatment. In addition, PCE ameliorated the MGO level, resulting in inhibition of the cytotoxic formation of stable adducts and $8-\mathrm{OHdG}$ expression (a known oxidative damage marker) in STZ-induced diabetic rats.

Severe hyperglycemia and podocyte loss is present at an early stage in STZ-induced diabetic animal models (29). Furthermore, podocyte damage correlates with worsening proteinuria and albuminuria (3). Previous studies have demonstrated that podocyte apoptosis is significant in the progression of glomerulosclerosis and proteinuria in diabetic nephropathy $(30,31)$. Therefore, there is an urgent requirement for the development of therapeutic agents for proteinuria and renal diseases, which act via direct podocyte targeting (32). Experimental and clinical studies have demonstrated podocyte loss, due to apoptosis, and that depletion leads to proteinuria in diabetic nephropathy $(5,33)$. Based on these results, it was hypothesized in the current study that PCE may preserve the podocyte cell number, ameliorate podocyte loss and reduce proteinuria. These protective effects were partly attributed to the anti-apoptotic action of PCE.

The effect of PCE on the levels of a key downstream effector of apoptosis, cleaved caspase-3 was investigated in the present study. Caspase- 3 is frequently activated by cell death processes, such as apoptosis and initiates apoptotic DNA fragmentation by proteolytic processing (34). In the current study, cleaved capase-3 expression levels were increased in the kidneys of STZ-induced diabetic rats. However, PCE treatment markedly reduced cleaved caspase-3 overexpression. The inhibitory effect of PCE on podocyte loss and apoptosis correlated with the reduction in caspase- 3 overexpression in the renal glomerular of STZ-induced diabetic rats. Thus, PCE may inhibit podocyte apoptosis in the kidneys of STZ-induced diabetic rats, in part, by reducing cleavage of caspase-3.

In addition, significantly increased $\mathrm{MGO}$ and $8-\mathrm{OHdG}$ levels were observed in STZ-induced diabetic rats. The formation of MGO is enhanced under diabetic conditions $(11,35)$. MGO is a known precursor of AGEs, and MGO-derived AGE levels have been found to be increased in diabetic renal tissue $(13,16)$. Furthermore, loss and depletion of glomerular cells as a result of apoptosis occurs in STZ-induced diabetic rats (36). Additionally, our previous study demonstrated that MGO is involved in podocyte loss and the expression of $8-\mathrm{OHdG}$ in podocytes of the diabetic kidney (16). In addition, the formation of $8-\mathrm{OHdG}$-adducted bases is associated with oxidative stress (37). When DNA is damaged by oxidative stress, cells elicit either apoptosis or cell cycle delay with apoptosis (38). MGO increases the intracellular oxidative stress level and enhances MGO accumulation, which is responsible for apoptotic damage (39). Thus, the results of the present study demonstrated that $\mathrm{MGO}$ and 8 -OHdG levels were increased in the kidney of STZ-induced diabetic rats, which was subsequently attenuated by PCE treatment.

$P$. cuspidatum contains flavones, such as resveratrol and emodin as the major chemical constituents $(27,28)$. Resveratrol is a natural antioxidant polyphenol that is present in $P$. cuspidatum, which exerts various biological functions, including anti-inflammatory and antioxidant activities $(40,41)$. In addition to antioxidant effects, resveratrol possesses additional properties that ameliorate diabetes or high glucose-induced kidney injury by activating 5'AMP-activated protein kinase or Sirtuin 1. Ding et al (42) reported that resveratrol treatment attenuates renal hypertrophy and urinary albumin excretion in the early stages of diabetes in STZ-induced diabetic rats, without affecting the blood glucose levels. In addition, resveratrol scavenges MGO and exerts inhibitory effects on AGE formation (25). Emodin performs various biological activities, such as antioxidant, anti-diabetic and anti-inflammatory 
activities (43-45). In particular, emodin exerts the protective effect of suppressing fibronectin and transforming growth factor- $\beta 1$ overexpression by inhibiting nuclear factor- $\kappa \mathrm{B}$ activation in diabetic nephropathy (46). Recently, emodin was demonstrated to affect antioxidant capacity and anti-apoptosis activity in hepatic cells, by directly affecting the mitochondria and acting against cytotoxicity (45). Notably, the present study revealed that emodin is a potent MGO scavenger and inhibited MGO-derived AGE formation, and its efficacy was markedly stronger than that of AG, a particularly well-known AGE inhibitor. These findings indicate that emodin and resveratrol are biologically active compounds of PCE, and the biological activities of PCE were attributed to the antioxidant and carbonyl scavenging capacity of these active ingredients. Although resveratrol and emodin are two major active chemicals of PCE, the primary active compound of PCE remains unclear. However, it is likely that treatment with PCE is effective against diabetic nephropathy, due to inhibition of apoptotic activity, which would promote renal podocyte loss, to the scavenging of the cytotoxic levels of MGO and oxidative stress for example.

In conclusion, these findings indicate that podocyte loss and proteinuria in STZ-induced diabetic rats were ameliorated by treatment with PCE, which was in part achieved by inhibiting podocyte apoptosis and cleaved caspase-3 expression, and by restoring the balance of MGO and oxidative stress. Therefore, PCE may serve as a valuable therapeutic strategy for the treatment of early diabetic nephropathy.

\section{Acknowledgements}

The present study was supported by grants from the KIOM (Daejeon, Korea: grant no. K14040).

\section{References}

1. White KE and Bilous RW: Type 2 diabetic patients with nephropathy show structural-functional relationships that are similar to type 1 disease. J Am Soc Nephrol 11: 1667-1673, 2000.

2. Steffes MW, Schmidt D, McCrery R, and Basgen JM; International Diabetic Nephropathy Study Group: Glomerular cell number in normal subjects and in type 1 diabetic patients. Kidney Int 59: 2104-2113, 2001.

3. Susztak K, Raff AC, Schiffer $M$ and Böttinger EP. Glucose-induced reactive oxygen species cause apoptosis of podocytes and podocyte depletion at the onset of diabetic nephropathy. Diabetes 55: 225-233, 2006.

4. Gui D, Guo Y, Wang F, Liu W, Chen J, Chen Y, Huang J and Wang N: Astragaloside IV, a novel antioxidant, prevents glucose-induced podocyte apoptosis in vitro and in vivo. PloS one 7: e39824, 2012.

5. Zhang Y, Chen B, Hou XH, Guan GJ, Liu G, Liu HY and Li XG: Effects of mycophenolate mofetil, valsartan and their combined therapy on preventing podocyte loss in early stage of diabetic nephropathy in rats. Chin med J 120: 988-995, 2007.

6. Estacio RO and Schrier RW: Diabetic nephropathy: Pathogenesis, diagnosis and prevention of progression. Adv Intern Med 46: 359-408, 2001

7. Ha H, Kim C, Son Y, Chung MH and Kim KH: DNA damage in the kidneys of diabetic rats exhibiting microalbuminuria. Free Radic Biol Med 16: 271-274, 1994.

8. Kakimoto M, Inoguchi T, Sonta T, Yu HY, Imamura M, Etoh $\mathrm{T}$, Hashimoto $\mathrm{T}$ and Nawata $\mathrm{H}$ : Accumulation of 8-hydroxy-2'-deoxyguanosine and mitochondrial DNA deletion in kidney of diabetic rats. Diabetes 51: 1588-1595, 2002.

9. Marshall CB,Pippin JW, Krofft RD and Shankland SJ: Puromycin aminonucleoside induces oxidant-dependent DNA damage in podocytes in vitro and in vivo. Kidney Int 70: 1962-1973, 2006.
10. Yamada H, Miyata S, Igaki N, Yatabe H, Miyauchi Y, Ohara T, Sakai M, Shoda H, Oimomi M and Kasuga M: Increase in 3-deoxyglucosone levels in diabetic rat plasma. Specific in vivo determination of intermediate in advanced Maillard reaction. J Biol Chem 269: 20275-20280, 1994.

11. McLellan AC, Thornalley PJ, Benn J and Sonksen PH: Glyoxalase system in clinical diabetes mellitus and correlation with diabetic complications. Clin Sci (Lond) 87: 21-29, 1994.

12. Lapolla A, Flamini R, Dalla Vedova A, Senesi A, Reitano R, Fedele D, Basso E, Seraglia R and Traldi P: Glyoxal and methylglyoxal levels in diabetic patients: Quantitative determination by a new GC/MS method. Clin Chem Lab Med 41: 1166-1173, 2003.

13. Randell EW, Vasdev S and Gill V: Measurement of methylglyoxal in rat tissues by electrospray ionization mass spectrometry and liquid chromatography. J Pharmacol Toxicol Methods 51: 153-157, 2005.

14. Amicarelli F, Colafarina S, Cattani F, Cimini A, Di Ilio C, Ceru MP and Miranda M: Scavenging system efficiency is crucial for cell resistance to ROS-mediated methylglyoxal injury. Free Radic Biol Med 35: 856-871, 2003

15. Pedchenko VK, Chetyrkin SV, Chuang P, Ham AJ, Saleem MA, Mathieson PW, Hudson BG and Voziyan PA: Mechanism of perturbation of integrin-mediated cell-matrix interactions by reactive carbonyl compounds and its implication for pathogenesis of diabetic nephropathy. Diabetes 54: 2952-2960, 2005.

16. Kim J, Sohn E, Kim CS and Kim JS: Renal podocyte apoptosis in Zucker diabetic fatty rats: Involvement of methylglyoxal-induced oxidative DNA damage. J Comp Pathol 144: 41-47, 2011.

17. Alarcon-Aguilara FJ, Roman-Ramos R, Perez-Gutierrez S, Aguilar-Contreras A, Contreras-Weber CC and Flores-Saenz JL: Study of the anti-hyperglycemic effect of plants used as antidiabetics. J Ethnopharmacol 61: 101-110, 1998.

18. Li WL, Zheng HC, Bukuru J and De Kimpe N: Natural medicines used in the traditional Chinese medical system for therapy of diabetes mellitus. J Ethnopharmacol 92: 1-21, 2004.

19. Ban SH, Kwon YR, Pandit S, Lee YS, Yi HK and Jeon JG: Effects of a bio-assay guided fraction from Polygonum cuspidatum root on the viability, acid production and glucosyltranferase of mutans streptococci. Fitoterapia 81: 30-34, 2010.

20. Bralley EE, Greenspan P, Hargrove JL, Wicker L and Hartle DK: Topical anti-inflammatory activity of Polygonum cuspidatum extract in the TPA model of mouse ear inflammation. J Inflamm (Lond) 5: 1, 2008.

21. Zhang H, Li C, Kwok ST, Zhang QW and Chan SW: A review of the pharmacological effects of the dried root of (hu zhang) and its constituents. Evid Based Complement Alternat Med 2013: 208349, 2013.

22. National Research Council: Guide for the Care and Use of Laboratory Animals. 8th edition. The National Academies Press, Washington, DC, USA, 2011.

23. Mundel P, Reiser J and Kriz W: Induction of differentiation in cultured rat and human podocytes. J Am Soc Nephrol 8: 697-705, 1997.

24. Schiffer M, Mundel P, Shaw AS and Böttinger EP: A novel role for the adaptor molecule $\mathrm{CD} 2$-associated protein in transforming growth factor-beta-induced apoptosis. J Biol Chem 279: 37004-37012, 2004.

25. Tang D, Zhu JX, Wu AG, Xu YH, Duan TT, Zheng ZG, Wang RS, Li D and Zhu Q: Pre-column incubation followed by fast liquid chromatography analysis for rapid screening of natural methylglyoxal scavengers directly from herbal medicines: Case study of Polygonum cuspidatum. J chromatogr A 1286: 102-110, 2013.

26. Shaheen F, Shmygol A, Rabbani N and Thornalley PJ: A fluorogenic assay for methylglyoxal. Bioche Soc Trans 42: 548-555, 2014.

27. Pan M and Wang X: Chemical constituents and pharmacological action of Polygonum cuspidatum Sieb. Et Zucc. Zhong Yao Cai 23: 56-58, 2000.

28. Qian G, Leung SY, Lu G and Leung KS: Differentiation of rhizoma et radix polygoni cuspidati from closely related herbs by HPLC fingerprinting. Chem Pharm Bull (Tokyo) 54: 1179-1186, 2006.

29. Siu B, Saha J, Smoyer WE, Sullivan KA and Brosius FC III: Reduction in podocyte density as a pathologic feature in early diabetic nephropathy in rodents: Prevention by lipoic acid treatment. BMC nephrol 7: 6, 2006.

30. RiedlE,PfisterF, Braunagel M, Brinkkötter P, Sternik P,Deinzer M, Bakker SJ, Henning RH, van den Born J, Krämer BK, et al: Carnosine prevents apoptosis of glomerular cells and podocyte loss in STZ diabetic rats. Cell Physiol Biochem 28: 279-288, 2011. 
31. Sohn E, Kim J, Kim CS, Kim YS, Jang DS and Kim JS: Extract of the aerial parts of Aster koraiensis reduced development of diabetic nephropathy via anti-apoptosis of podocytes in streptozotocin-induced diabetic rats. Biochem Biophys Res Commun 391: 733-738, 2010.

32. Reiser J, Gupta V and Kistler AD: Toward the development of podocyte-specific drugs. Kidney Int 77: 662-668, 2010.

33. Meyer TW, Bennett PH and Nelson RG: Podocyte number predicts long-term urinary albumin excretion in Pima Indians with Type II diabetes and microalbuminuria. Diabetologia 42: 1341-1344, 1999.

34. Nuñez G, Benedict MA, Hu Y and Inohara N: Caspases: The proteases of the apoptotic pathway. Oncogene 17: 3237-3245, 1998.

35. Oya T, Hattori N, Mizuno Y, Miyata S, Maeda S, Osawa T and Uchida K: Methylglyoxal modification of protein. Chemical and immunochemical characterization of methylglyoxal-arginine adducts. J Biol Chem 274: 18492-18502, 1999.

36. Pesce C, Menini S, Pricci F, Favre A, Leto G, DiMario U and Pugliese G: Glomerular cell replication and cell loss through apoptosis in experimental diabetes mellitus. Nephron 90: 484-488, 2002.

37. Li CS, Wu KY, Chang-Chien GP and Chou CC: Analysis of oxidative DNA damage 8-hydroxy-2'-deoxyguanosine as a biomarker of exposures to persistent pollutants for marine mammals. Environ Sci Technol 39: 2455-2460, 2005.

38. Zgheib O, Huyen Y, DiTullio RA Jr, Snyder A, Venere M, Stavridi ES and Halazonetis TD: ATM signaling and 53BP1. Radiother Oncol 76: 119-122, 2005.

39. Kim OS, Kim J, Kim CS, Kim NH and Kim JS: KIOM-79 prevents methyglyoxal-induced retinal pericyte apoptosis in vitro and in vivo. J Ethnophamacol 129: 285-292, 2010.
40. Ikizler M, Ovali C, Dernek S, Erkasap N, Sevin B, Kaygisiz Z and Kural T: Protective effects of resveratrol in ischemia-reperfusion injury of skeletal muscle: A clinically relevant animal model for lower extremity ischemia. Chin J Physiol 49: 204-209, 2006.

41. Kong LD, Yang C and Qiu X: Effects of processing on antioxidation of radix et rhizoma rhei and rhizoma polygoni cuspidati. Zhongguo Zhong Yao Za Zhi 26: 388-391, 2001 (In Chinese).

42. Ding DF, You N, Wu XM, Xu JR, Hu AP, Ye XL, Zhu Q, Jiang XQ, Miao H, Liu C and Lu YB: Resveratrol attenuates renal hypertrophy in early-stage diabetes by activating AMPK. Am J Nephrol 31: 363-374, 2010.

43. Lu Y, Jeong YT, Li X, Kim MJ, Park PH, Hwang SL, Son JK and Chang HW: Emodin isolated from polygoni cuspidati radix inhibits tnf-alpha and il-6 release by blockading nf-kappab and map kinase pathways in mast cells stimulated with PMA Plus A23187. Biomol Thera 21: 435-441, 2013.

44. Yang J, Zeng Z, Wu T, Yang Z, Liu B and Lan T: Emodin attenuates high glucose-induced TGF- $\beta 1$ and fibronectin expression in mesangial cells through inhibition of NF- $\mathrm{B}$ pathway. Exp Cell Res 319: 3182-3189, 2013.

45. Cui YT, Liu B, Xie J, Xu P, Habte-Tsion HM and Zhang YY: The effect of emodin on cytotoxicity, apoptosis and antioxidant capacity in the hepatic cells of grass carp (Ctenopharyngodon idellus). Fish Shellfish Immunol 38: 74-79, 2014.

46. Li X, Liu W, Wang Q, Liu P, Deng Y, Lan T, Zhang X, Qiu B, Ning $\mathrm{H}$ and Huang $\mathrm{H}$ : Emodin suppresses cell proliferation and fibronectin expression via p38MAPK pathway in rat mesangial cells cultured under high glucose. Mol Cell Endocrinol 307: 157-162, 2009. 